JBR, Note, revised on $25^{\text {th }}$ April 2016

J. Breath Res. 10 (2016) 021002 10.1088/1752-7155/10/2/021002

\title{
Breath concentration of acetic acid vapour is elevated in patients with cystic fibrosis.
}

David Smith ${ }^{1}$, Kristýna Sovová ${ }^{2}$, Kseniya Dryahina ${ }^{2}$, Tereza Doušová ${ }^{3}$, Pavel Dřevínek ${ }^{4}$, Patrik Španěl ${ }^{2 *}$

${ }^{1}$ Institute for Science and Technology in Medicine, Medical School, Keele University, Thornburrow Drive, Hartshill, Stoke-on-Trent ST4 7QB, UK

${ }^{2}$ J. Heyrovský Institute of Physical Chemistry, Academy of Sciences of the Czech Republic, v.v.i., Dolejškova 3, 18223 Prague 8, Czech Republic

${ }^{3}$ Department of Paediatrics, 2nd Faculty of Medicine, Charles University in Prague and Motol University Hospital, V Uvalu 84, 15006, Prague 5, Czech Republic

${ }^{4}$ Department of Medical Microbiology 2nd Faculty of Medicine, Charles University in Prague and Motol University Hospital, V Uvalu 84, 15006, Prague 5, Czech Republic

*Corresponding author

Professor P. Španěl

E-mail: spanel@jh-inst.cas.cz

\begin{abstract}
A study has been carried out of the volatile organic compounds present in the exhaled breath of 58 cystic fibrosis (CF) patients. An important observation is that the acetic acid vapour concentration measured by selected ion flow tube mass spectrometry, SIFT-MS, is significantly elevated in the exhaled breath of CF patients, independent of the Pseudomonas aeruginosa (PA) infection status (PA-infected median 170 ppbv; PA-negative median 182 ppbv), compared to that of healthy controls (median $48 \mathrm{ppbv}$ ). The cause for this may be decreased $\mathrm{pH}$ of the mucus lining the CF airways. Thus, we speculate that non-invasive measurement of breath acetic acid concentration could serve as an indicator of the acidity of the CF airways mucosa.
\end{abstract}




\section{Introduction}

Exhaled breath analysis as a potential non-invasive support to clinical diagnosis and therapeutic monitoring is a growing area of research that is showing great promise. In this regard, the search is on for volatile metabolites that are reliable indicators of respiratory infection and systemic disease. The relevant metabolites are mostly volatile organic compounds, VOCs, but also include some inorganic compounds such as nitric oxide and ammonia. [1]. Selected ion flow tube mass spectrometry, SIFT-MS (see concise overviews in [2-4]), allows the analysis of exhaled breath to be accomplished both in real time and on-line in single breath exhalations or of breath samples collected into bags for off-line analysis. Using SIFT-MS breath analysis has been accomplished of significantly large cohorts of healthy controls and thus the establishment of reference ranges of the most common breath metabolites have been achieved [5].

A particular aspect of SIFT-MS breath analysis research is directed towards identification of volatile biomarkers of respiratory bacteria. Much effort has been given to Pseudomonas aeruginosa (PA) that commonly infect the lungs of cystic fibrosis (CF) patients, which has involved detailed studies of in vitro cultures of PA, including many genetically identified strains and their biofilm or planktonic modes of growth. This work, over a decade, has resulted in the establishment of hydrogen cyanide, HCN, as a volatile biomarker of PA [6-9]. Most recently, a multi-centre study involving $8 \mathrm{UK}$ hospitals has quantified HCN in the exhaled breath of 233 children with CF and demonstrates that HCN is indeed a discreet specific (albeit not yet entirely sensitive) biomarker of PA. The exciting results of this SPACE study are reported recently in ERJ Open Research [10].

The pathology of CF is well documented. It is a genetically inherited, life-shortening chronic illness associated with excessive abnormal mucus production in the airways and breathing becomes increasingly difficult. The lungs then become a rich nutrient medium for opportunistic pathogens that are normally harmless for healthy people but cannot be removed fast enough in CF thus encouraging chronic respiratory infections such as PA, Staphylococcus aureus (SA) [11, 12] and Burkholderia cepacia [13, 14] and the fungus Aspergillus fumigatus (AF) [15]. Currently, the detection of these pathogens in the airways is achieved by culturing sputum samples, but CF children have difficulty in expectorating sputum; hence the attraction of non-invasive breath analysis to detect volatile compound biomarkers of specific pathogens.

Continuing with this research, we have carried out a study of the VOCs in the exhaled breath of two groups of CF patients, one whose airways are infected with PA and those who are PA negative. The major question posed was whether or not other volatile compounds in the breath, other than HCN, can be used to distinguish between these CF patient groups. The detailed results of this wider study will be published in an upcoming paper (Španěl et. al., in preparation). However, the focus of the present note is to report the serendipitous observation of greatly elevated absolute concentrations of acetic acid vapour in the exhaled breath of the CF patients compared to those measured for healthy persons. This surprising and unexpected observation may have relevance to the physiology in CF and its clinical treatment. This note thus presents the detailed experimental data for acetic acid and compares these results with the results for healthy cohorts, and briefly discusses the origin of the acetic acid and its implications in $\mathrm{CF}$. 


\section{Materials and methods}

\section{Subjects}

A total of 58 patients with CF (26 males and 32 females, age range 2-41 years, median 14 years) were recruited at the Prague Motol University Hospital. Of these, 38 were assessed as PA negative and 20 were infected with PA (according to a positive result of a PCR test, in 18 patients the diagnosis was confirmed by cultivation). The study was approved by the institutional ethics committee and the patients or their legal guardians provided an appropriate written consent.

Breath analysis.

Mixed expiratory breath samples were collected in the morning (before 12:00) during regular visits by the patients to the CF clinic. Each patient was asked to slowly inflate a $4 \mathrm{~L}$ disposable Nalophan bag through an $8 \mathrm{~cm}$ long PTFE tube (1/4 inch OD, 0.23 inch ID), which requires several exhalations. The typical flow rate was about $100 \mathrm{~mL} / \mathrm{s}$ as restricted by the ID of the PTFE tube. The sample bags were then immediately placed into a temperature controlled enclosure held at $37^{\circ} \mathrm{C}$ and coupled to the SIFT-MS instrument via a heated calibrated capillary. The instrument was operated in the multiple ion monitoring mode to acquire the count rates of the reagent and analyte ions at all integral values of $m / z$ within the range 10 to 120. Analysis was performed within 20 minutes to avoid diffusive losses through the bag material, although previous studies have shown that such losses are not significant for wide-ranging compound types over a storage period of 24 hours. (see [16]). The room air was routinely analysed prior to coupling the sample bag to the SIFT-MS instrument. For GC-MS analysis of the same sample, an SPME fibre was inserted into the bag for $30 \mathrm{~min}$ at the enclosure temperature of $37^{\circ} \mathrm{C}$.

\section{SIFT-MS quantification and identification of acetic acid}

Both positive identification and accurate quantification of volatile metabolites in breath is essential for their concentrations to be used as disease biomarkers. Thus, for SIFT-MS analysis of any trace compound present in humid exhaled breath, it is important to understand the underpinning ion chemistry. The principles and operation of SIFT-MS need not be described in detail here, since they are well established [2, $\underline{3}, \underline{17}, \underline{18}]$. It is sufficient to say that SIFT-MS exploits controlled chemical ionization of trace compounds in air/breath samples by the reagent ions $\mathrm{H}_{3} \mathrm{O}^{+}, \mathrm{NO}^{+}$and $\mathrm{O}_{2}{ }^{+\bullet}$, either individually or in combinations, in a helium-buffered flow tube and the characteristic analyte ions produced are identified and counted by the downstream analytical mass spectrometer/detection system. Concentrations of the neutral trace analyte molecules in the sample are then calculated $[\underline{17}, \underline{18}]$. The most suitable reagent ion for analyses of acetic acid in the vapour phase is $\mathrm{NO}^{+}$, as has been established in previous studies [19-21]. $\mathrm{NO}^{+}(\mathrm{m} / \mathrm{z} 30)$ reacts with $\mathrm{CH}_{3} \mathrm{COOH}$ under SIFT-MS reactor conditions to form an analyte adduct ion as follows:

$$
\mathrm{NO}^{+}+\mathrm{CH}_{3} \mathrm{COOH}+\mathrm{He} \rightarrow \mathrm{NO}^{+} . \mathrm{CH}_{3} \mathrm{COOH}+\mathrm{He}
$$


The helium atom acts only as a mediator to stabilise the excited adduct analyte ion $\left(\mathrm{NO}^{+} . \mathrm{CH}_{3} \mathrm{COOH}\right)^{*}(\mathrm{~m} / \mathrm{z}$ 90) against unimolecular dissociation back to the ion/molecule reactants. Then the monohydrate of the adduct ion, $\mathrm{NO}^{+} . \mathrm{CH}_{3} \mathrm{COOH} . \mathrm{H}_{2} \mathrm{O}(\mathrm{m} / \mathrm{z} 108)$, is similarly formed in the presence of water vapour molecules introduced into the helium carrier gas of the SIFT-MS reactor when a sample of very humid exhaled breath is being analysed. This monohydrate ion must be included as an additional analyte ion in order to obtained accurate SIFT-MS analysis of acetic acid. Thus, the acetic acid concentration was calculated from the count rates of reagent ions at $\mathrm{m} / \mathrm{z} 30,48,66$ and analyte ions at $\mathrm{m} / \mathrm{z} 90$ and 108 . What is equally important is that previous work has shown that the ratio of the signal levels of the adduct ion $(\mathrm{m} / \mathrm{z} 90)$ and its monohydrate $(\mathrm{m} / \mathrm{z} 108)$ at the downstream analytical mass spectrometer depends on the water vapour concentration in the breath sample [20], which is close to $6 \%$ by volume at the donor body temperature (36-37C) and which is routinely measured during SIFT-MS analyses [22]. Thus, the measurement of the $\mathrm{m} / \mathrm{z} 108$ to $(\mathrm{m} / \mathrm{z} 90+$ $\mathrm{m} / \mathrm{z}$ 108) signal ratio acts as an important check on the proper identification of the acetic acid.

\section{GC-MS confirmation of the presence of acetic acid}

Solid phase microextraction (SPME) was used to identify trace compounds present in these bag samples by GC-MS analyses. Carboxene polydimethylsiloxane (CAR/PDMS)-coated SPME fibres (Supelco, Bellefonte, PA, USA) were inserted into the bags for $30 \mathrm{~min}$ at a temperature of $37^{\circ} \mathrm{C}$. The fibres were directly inserted into the injector (heated to $220^{\circ} \mathrm{C}$ ) of the GC-MS instrument (FOCUS GC with ITQ 700 ion trap mass spectrometer, Thermo Fisher Scientific Inc., USA). The GC oven temperature program was the following: $38^{\circ} \mathrm{C}$ (hold $8 \mathrm{~min}$ ), $4^{\circ} \mathrm{C} / \mathrm{min}$ ramp up to $100{ }^{\circ} \mathrm{C}, 30^{\circ} \mathrm{C} / \mathrm{min}$ ramp up to $210^{\circ} \mathrm{C}$, and a final hold 3 min (total run time $30 \mathrm{~min}$ ). The GC capillary column used was a TG-624 (Thermo Fisher Scientific Inc., USA) with helium carrier gas flow of $1 \mathrm{~mL} / \mathrm{min}$. Electron ionisation $(70 \mathrm{eV})$ was used to analyse the eluting molecules by the ion trap operating in the scan mode $(\mathrm{m} / \mathrm{z}$ 400, scan rate $1 \mathrm{scan} / \mathrm{s}$ ). Peak identification was based on mass spectral interpretation and comparisons with the NIST 2.0 library.

A sample chromatogram and a mass spectrum confirming the presence of acetic acid is shown in Figure 1. By this GC-MS and SIFT-MS combined approach we can be confident that we are identifying and quantifying correctly the acetic acid in exhaled breath.

\section{Results}

The present measurements of the acetic acid breath concentrations for all the CF patients enrolled into the study are given in Figure 2, as a box-and whisker plot. It is very important to note that no significant difference was observed between the breath acetic acid concentrations of the PA-infected (median 170, 110-210 ppbv) and the PA-negative groups (median 182; IQR 139-336 ppbv). However, these concentrations are several times greater than endexpiratory concentration of acetic acid established for the healthy population (median 48 ppbv, range 20-60 ppbv, see Figure 2). [23]. It is pertinent to note that the concentrations of acetic acid present in the ambient air were routinely checked by SIFT-MS analyses of the room air and no measurable background was observed.

\section{Discussion}


Until recently, acetic acid received little attention as an endogenous compound, but it has been known for some time to be present in exhaled breath condensate [24] and saliva [24, 25]. Acetate, the anionic form of acetic acid in biological fluids, is a physiological metabolite involved in normal human biochemistry. Plasma acetate concentrations in healthy people

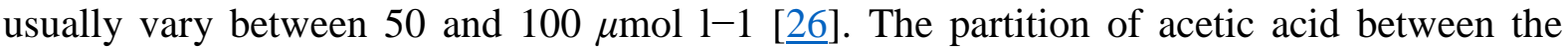
gaseous and liquid concentration will be strongly influenced by $\mathrm{pH}$ of the solution phase and can be estimated according to the Henry's Law constant for this fatty acid [27]. It turns out that for the above acetate concentration and for a $\mathrm{pH}$ of the airways mucosa near to 7 , the exhaled breath concentration of acetic acid should be of the order of 10 parts-per-billion by volume ppbv [23]. At lower pH (acidic mucosa) the gas phase (exhaled breath) acetic acid will correspondingly be increased.

The first SIFT-MS analysis of breath acetic acid followed our research of the ion chemistry required for identifying and quantifying the isobaric compounds propanol, methyl formate and acetic acid by SIFT-MS [20]. Thus, the ion-molecule kinetics were established for the accurate analysis of acetic acid using $\mathrm{NO}^{+}$reagent ions. In this study the concentration of acetic acid in mouth-exhaled breath samples from five healthy volunteers collected into Nalophan bags using the same procedure as used in the present study was measured using SIFT-MS and was seen to vary from about 30-60 ppbv and the concentrations in noseexhaled breath were about a factor of 2 lower. That the oral cavity is a significant source of acetic acid was also demonstrated by analysing the oral cavity static gas after a mouth wash with water, when the concentrations reduced to about $10 \mathrm{ppb}$ showing that this compound is also partially systemic, i.e., originating at the alveolar interface and/or in the airways mucosa. It is worthy of note that fatty acids have been detected in exhaled breath from healthy persons by direct analysis using electrospray ionization and atmospheric pressure ionizationmass spectrometry [28, 29] A further relevant observation is that when analysing the exhaled breath of a very limited cohort of patients with lung tumours by SIFT-MS, it was observed that breath acetic acid was elevated above that for breath healthy controls and we tentatively argued that this may be because the tissue surrounding the lung tumours was at a lower $\mathrm{pH}$ than healthy lung tissue [30].

More relevant to the present results is a previous concerted study to identify potential biomarkers in the breath of patients suffering from gastro-oesophageal reflux disease (GERD) using both SIFT-MS and GC-MS analysis in parallel to ensure positive identification of VOCs [23]. End-expiratory concentration of acetic acid measured by SIFTMS in mouth exhaled breath of 22 GERD patients (median 85 ppbv, see Figure 2) was found to be significantly higher than that in breath of a control group of 24 healthy persons (median 48 ppbv). This increase of acetic acid in GERD may be due to locally decreased $\mathrm{pH}$ of the surface of the airways/trachea. It was concluded that breath acetic acid may be useful for noninvasive diagnostics of GERD.

However, the present results clearly reveal that breath acetic acid is greatly elevated in CF patients above both healthy controls and the GERD patients; the median value is a factor of 2 higher than those for the GERD patients and about 4 times higher than the healthy group. Yet there is acetic acid at the very large concentrations of $1100 \mathrm{ppbv}$ and $1600 \mathrm{ppbv}$ in two of the CF patients (see Figure 2); these concentrations which were very thoroughly checked and 
verified are 30-40 times greater than normal. This is a unique differentiation between healthy and the diseased patients as far as breath analysis of volatile biomarkers is concerned and deserves a physiological explanation because it may have implications to the diagnosis and treatment of CF.

Since there is no significant difference in breath acetic acid concentration between the PAinfected and PA-free groups, the conclusion must be that this acid is not produced by the PA bacteria. So what is its origin? Is it due to the abnormal mucus congestion in the lungs and airways of the CF patients and is the mucus at a low $\mathrm{pH}$ (acidic) relative to normal respiratory track tissue? If this is so, it would effectively move the equilibrium from liquid phase acetate ions (non-volatile) to gas phase acetic acid molecules (volatile).

There have been discussions and conflicting ideas as to the $\mathrm{pH}$ of the mucosa in the CF lung and airways [31], some clinicians suggesting that therapy may be directed toward increasing the $\mathrm{pH}$ of airways [32]. Our results apparently suggest that the mucosa in CF is acidic given the efficient release of acetic acid vapour. Support for an acidic mucosa comes also from a recent study of the new-born porcine lung [33] in which it was found that at birth these lungs lack infection and inflammation, but have a reduced ability to eradicate bacteria. In the healthy pigs the thin layer of airway surface liquid rapidly killed bacteria in vivo. However, it was found that the mucosa $\mathrm{pH}$ was more acidic in the CF pigs, which inhibited the antimicrobial activity of the mucosa. The increased acidity was linked to a channel that facilitates $\mathrm{HCO}_{3}-$ anion production and impairs the killing of bacteria that enter the newborn lung. These findings again indicate that increasing mucosa $\mathrm{pH}$ might prevent the initial infection in patients with CF. It is also pertinent to recognise that that the $\mathrm{pH}$ of exhaled breath condensate was found to be significantly lower in CF subjects ( $\mathrm{pH}$ 6.6) compared to that in a control group ( $\mathrm{pH}$ 8.1) [34].

\section{Conclusion}

The data presented in this note are the first observation and measurement of elevated concentrations of acetic acid in the exhaled breath of CF patients. Thus, we speculate that breath acetic acid could be a non-invasive biomarker of the acidity of the airways mucosa, which could help to assess the risk of bacterial infection and ultimately improve the efficacy of CF therapy. It may be that measurement of acetic acid in exhaled breath could support CF diagnosis alongside the standard sweat test. Clearly, further work is needed to substantiate these findings using greater cohorts of healthy volunteers and CF patients.

\section{Acknowledgements}

We gratefully acknowledge funding from Czech Science Foundation GACR project No 14-14534S.

\section{References}

[1] Amann A and Smith D 2013 Volatile biomarkers: Non-Invasive Diagnosis in Physiology and Medicine Boston: Elsevier)

[2] Smith D and Španěl P 2015 The SIFT and FALP techniques; applications to ionic and electronic reactions studies and their evolution to the SIFT-MS and FA-MS analytical methods Int. $J$. Mass Spectrom. 377 467-78 
[3] Španěl P and Smith D 2011 Progress in SIFT-MS: Breath analysis and other applications Mass Spectrom. Rev. 30 236-67

[4] Smith D, Španěl P, Herbig J and Beauchamp J 2014 Mass spectrometry for real-time quantitative breath analysis J. Breath Res. 8027101

[5] Smith D, Turner C and Španěl P 2007 Volatile metabolites in the exhaled breath of healthy volunteers: their levels and distributions J. Breath Res. 1014004

[6] Smith D, Španěl P, Gilchrist F J and Lenney W 2013 Hydrogen cyanide, a volatile biomarker of Pseudomonas aeruginosa infection J. Breath Res. 7044001

[7] Gilchrist F J, Bright-Thomas R J, Jones A M, Smith D, Španěl P, Webb A K and Lenney W 2013 Hydrogen cyanide concentrations in the breath of adult cystic fibrosis patients with and without Pseudomonas aeruginosa infection J. Breath Res. 7026010

[8] Gilchrist F J, Sims H, Alcock A, Belcher J, Jones A M, Smith D, Španěl P, Webb A K and Lenney W 2012 Quantification of hydrogen cyanide and 2-aminoacetophenone in the headspace of Pseudomonas aeruginosa cultured under biofilm and planktonic conditions Analytical Methods 4 3661-5

[9] Gilchrist F J, Alcock A, Belcher J, Brady M, Jones A, Smith D, Španěl P, Webb K and Lenney W 2011 Variation in hydrogen cyanide production between different strains of Pseudomonas aeruginosa European Respiratory Journal 38 409-14

[10] Gilchrist F J, Belcher J, Jones A M, Smith D, Smyth A R, Southern K W, Španěl P, Webb A K and Lenney W 2015 Exhaled breath hydrogen cyanide as a marker of early Pseudomonas aeruginosa infection in children with cystic fibrosis ERJ Open Research 1

[11] Gilchrist F J, Spanel P, Smith D and Lenney W 2015 The in vitro identification and quantification of volatile biomarkers released by cystic fibrosis pathogens Analytical Methods 7 81824

[12] Chippendale T W E, Gilchrist F J, Španěl P, Alcock A, Lenney W and Smith D 2014 Quantification by SIFT-MS of volatile compounds emitted by in vitro cultures of S. aureus, S. pneumoniae and $\mathrm{H}$. influenzae isolated from patients with respiratory diseases Analytical Methods 6 2460-72

[13] Gilchrist F J, Sims H, Alcock A, Jones A M, Bright-Thomas R J, Smith D, Španěl P, Webb A K and Lenney W 2013 Is Hydrogen Cyanide a Marker of Burkholderia cepacia Complex? Journal of Clinical Microbiology 51 3849-51

[14] Shestivska V, Dryahina K, Nunvář J, Sovová K, Elhottová D, Smith D and Španěl P 2015 Quantitative analysis of volatile metabolites released in vitro by bacteria of the genus Stenotrophomonas for identification of breath biomarkers of respiratory infection in cystic fibrosis Jounal of Breath Research 9027104

[15] Chippendale T W E, Gilchrist F J, Španěl P, Alcock A, Lenney W and Smith D 2014 Quantification by SIFT-MS of volatile compounds emitted by Aspergillus fumigatus cultures and in co-culture with Pseudomonas aeruginosa, Staphylococcus aureus and Streptococcus pneumoniae Analytical Methods 6 8154-64

[16] Ghimenti S, Lomonaco T, Bellagambi F G, Tabucchi S, Onor M, Trivella M G, Ceccarini A, Fuoco R and Francesco F D 2015 Comparison of sampling bags for the analysis of volatile organic compounds in breath J. Breath Res. 9047110

[17] Španěl P, Dryahina K and Smith D 2006 A general method for the calculation of absolute trace gas concentrations in air and breath from selected ion flow tube mass spectrometry data Int. J. Mass Spectrom. 249 230-9

[18] Španěl P and Smith D 2013 Advances in On-line Absolute Trace Gas Analysis by SIFT-MS Curr. Anal. Chem. 9 525-39

[19] Michalčíková R B and Španěl P 2014 A selected ion flow tube study of the ion molecule association reactions of protonated $\left(\mathrm{MH}^{+}\right)$, nitrosonated $\left(\mathrm{MNO}^{+}\right)$and dehydroxidated $(\mathrm{M}-\mathrm{OH})^{(+)}$ carboxylic acids (M) with $\mathrm{H}_{2} \mathrm{O}$ Int. J. Mass Spectrom. 368 15-22

[20] Pysanenko A, Španěl P and Smith D 2009 Analysis of the isobaric compounds propanol, acetic acid and methyl formate in humid air and breath by selected ion flow tube mass spectrometry, SIFT-MS Int. J. Mass Spectrom. 285 42-8 
[21] Španěl P and Smith D 1998 SIFT studies of the reactions of $\mathrm{H}_{3} \mathrm{O}^{+}, \mathrm{NO}^{+}$and $\mathrm{O}_{2}{ }^{+}$with a series of volatile carboxylic acids and esters Int. J. Mass Spectrom. 172 137-47

[22] Španěl P and Smith D 2001 On-line measurement of the absolute humidity of air, breath and liquid headspace samples by selected ion flow tube mass spectrometry Rapid Commun. Mass Spectrom. 15 563-9

[23] Dryahina K, Pospisilova V, Sovova K, Shestivska V, Kubista J, Spesyvyi A, Pehal F, Turzikova J, Votruba J and Spanel P 2014 Exhaled breath concentrations of acetic acid vapour in gastro-esophageal reflux disease J. Breath Res. 8037109

[24] Effros R M, Casaburi R, Su J, Dunning M, Torday J, Biller J and Shaker R 2006 The effects of volatile salivary acids and bases on exhaled breath condensate $\mathrm{pH}$ Am. J. Respir. Crit. Care Med. 173 386-92

[25] Chen Z F, Darvell B W and Leung V W H 2004 Human salivary anionic analysis using ion chromatography Arch. Oral Biol. 49 863-9

[26] Tollinger C D, Vreman H J and Weiner M W 1979 Measurement of acetate in human blood by gas chromatography: effects of sample preparation, feeding, and various diseases Clin Chem 25 1787-90

[27] Sander R 1999 Compilation of Henry's Law constants for inorganic and organic species of potential importance in environmental chemistry Mainz: Max-Planck Institute of Chemistry)

[28] Martinez-Lozano P and de la Mora J F 2008 Direct Analysis of Fatty Acid Vapors in Breath by Electrospray Ionization and Atmospheric Pressure Ionization-Mass Spectrometry Anal. Chem. 80 8210-5

[29] Martinez-Lozano P, Zingaro L, Finiguerra A and Cristoni S 2011 Secondary electrospray ionization-mass spectrometry: breath study on a control group J. Breath Res. 5016002

[30] Smith D, Španěl P and Sule-Suso J 2010 Advantages of breath testing for the early diagnosis of lung cancer Expert Review of Molecular Diagnostics 10 255-7

[31] McShane D, Davies J C, Davies M G, Bush A, Geddes D M and Alton E W F W 2003 Airway surface $\mathrm{pH}$ in subjects with cystic fibrosis European Respiratory Journal 21 37-42

[32] Garland A L, Walton W G, Coakley R D, Tan C D, Gilmore R C, Hobbs C A, Tripathy A, Clunes L A, Bencharit S, Stutts M J, Betts L, Redinbo M R and Tarran R 2013 Molecular basis for $\mathrm{pH}$-dependent mucosal dehydration in cystic fibrosis airways Proceedings of the National Academy of Sciences of the United States of America 110 15973-8

[33] Pezzulo A A, Tang X X, Hoegger M J, Abou Alaiwa M H, Ramachandran S, Moninger T O, Karp P H, Wohlford-Lenane C L, Haagsman H P, van Eijk M, Banfi B, Horswill A R, Stoltz D A, McCray P B, Welsh M J and Zabner J 2012 Reduced airway surface pH impairs bacterial killing in the porcine cystic fibrosis lung Nature 487 109-13

[34] Newport S, Amin N and Dozor A J 2009 Exhaled breath condensate pH and ammonia in cystic fibrosis and response to treatment of acute pulmonary exacerbations Pediatr. Pulmonol. 44 866-72 

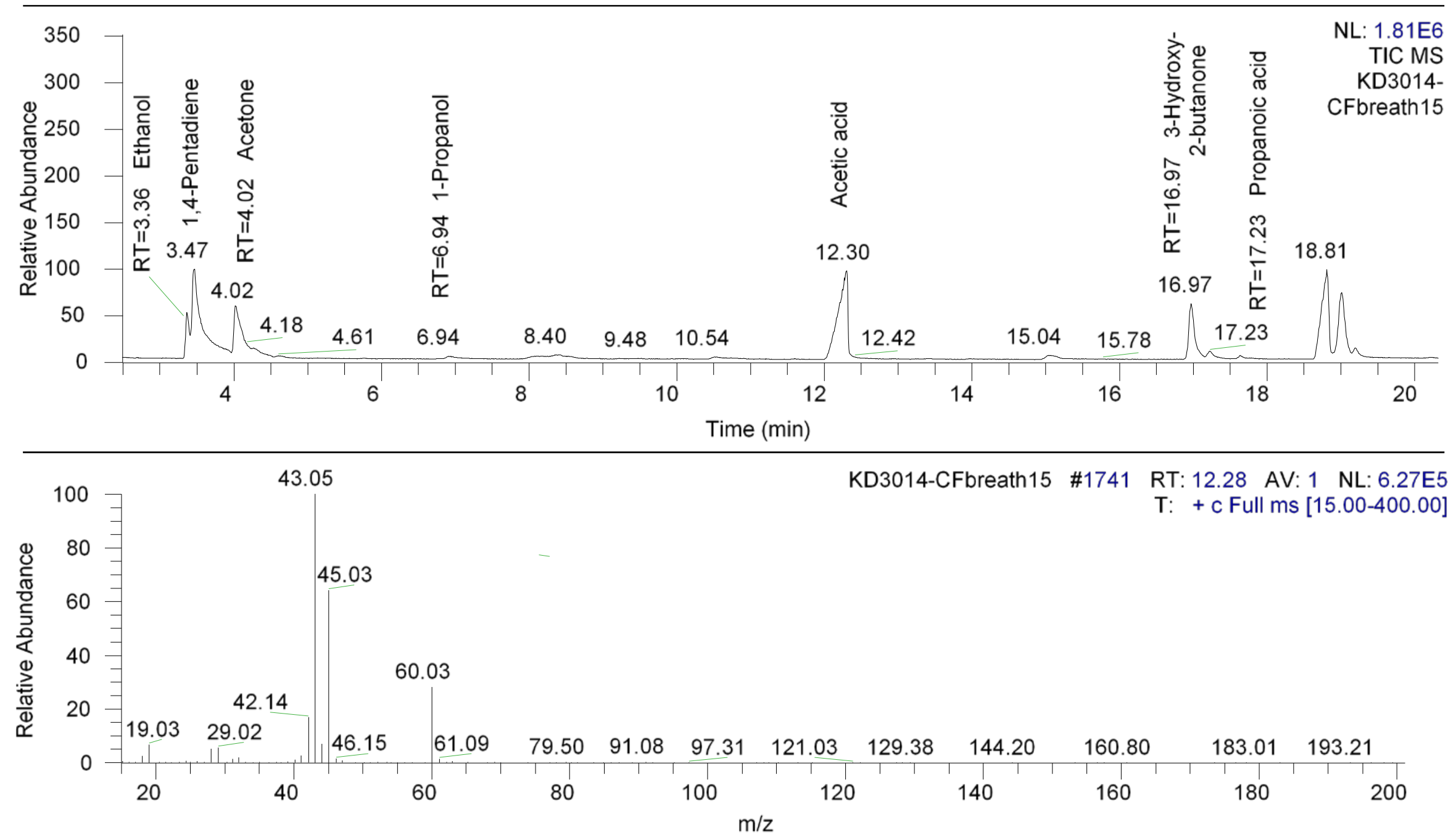

Figure 1. The GC-MS chromatogram of SPME-extracted volatile compound from a breath sample of one of the CF patients confirming the presence of acetic acid by the elution time and the ion fragmentation spectrum. 


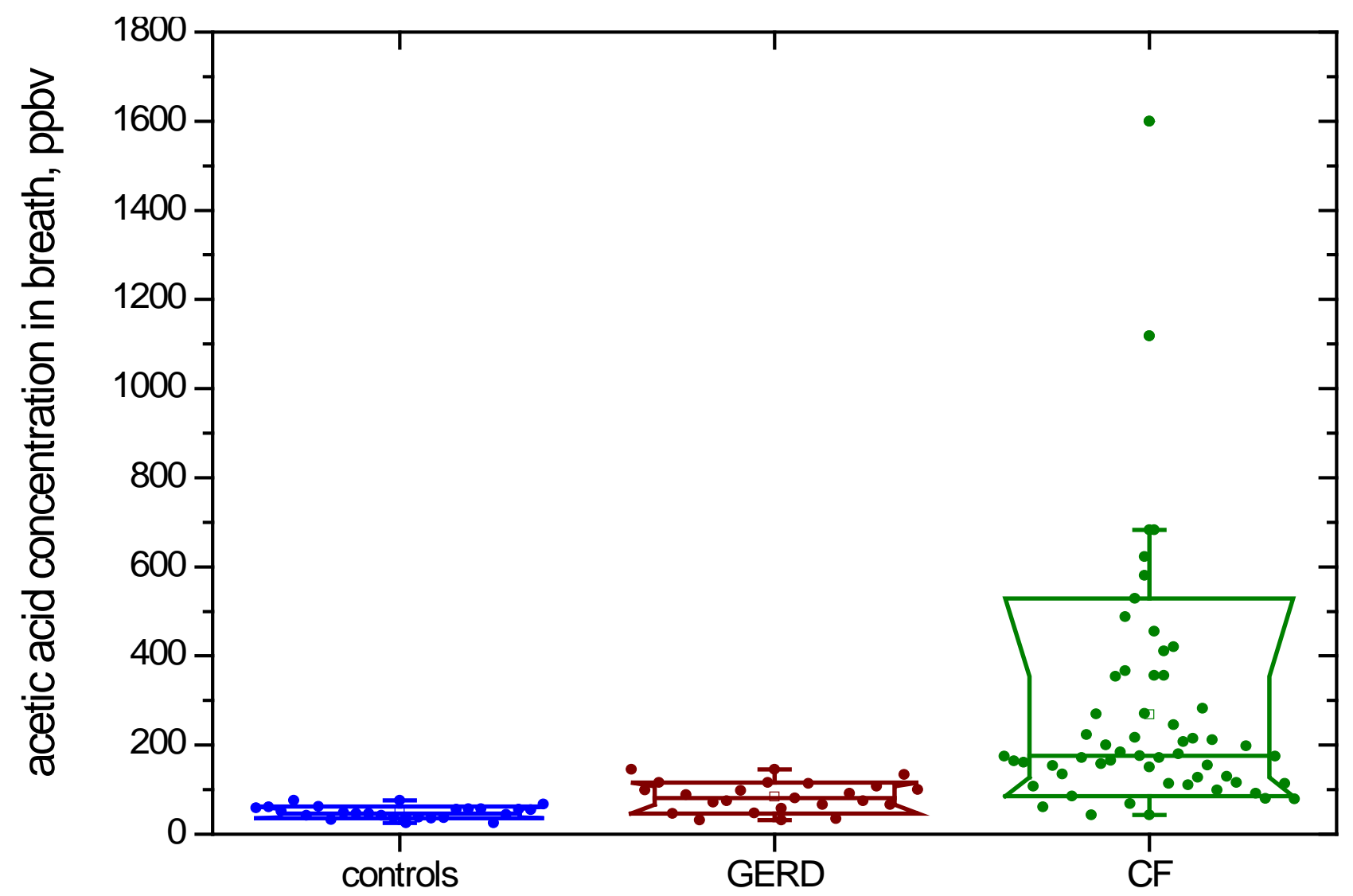

Figure 2 Concentrations of acetic acid in exhaled breath of healthy controls and patients with GERD (from ref. 24) and patients with CF obtained using SIFTMS. In addition to the individual data points the box-and-whisker plots are included to indicate the 10th, 25th, 50th, 75th and 90th percentiles. 